\title{
OPEN Diplazium esculentum (Retz.) Sw. reduces BACE-1 activities and amyloid peptides accumulation in Drosophila models of Alzheimer's disease
}

Thanit Kunkeaw ${ }^{1}$, Uthaiwan Suttisansanee ${ }^{1}$, Dunyaporn Trachootham ${ }^{1}$, Jirarat Karinchai ${ }^{2}$, Boonrat Chantong ${ }^{3}$, Saranyapin Potikanond ${ }^{4}$, Woorawee Inthachat ${ }^{1}$, Pornsiri Pitchakarn ${ }^{2}$ \& Piya Temviriyanukul ${ }^{1 凶}$

Alzheimer's disease (AD), one type of dementia, is a complex disease affecting people globally with limited drug treatment. Thus, natural products are currently of interest as promising candidates because of their cost-effectiveness and multi-target abilities. Diplazium esculentum (Retz.) Sw., an edible fern, inhibited acetylcholinesterase in vitro, inferring that it might be a promising candidate for $A D$ treatment by supporting cholinergic neurons. However, evidence demonstrating anti-AD properties of this edible plant via inhibiting of neurotoxic peptides production, amyloid beta (A $\beta$ ), both in vitro and in vivo is lacking. Thus, the anti-AD properties of $D$. esculentum extract both in vitro and in Drosophila models of $A \beta$-mediated toxicity were elucidated. Findings showed that an ethanolic extract exhibited high phenolics and flavonoids, contributing to antioxidant and inhibitory activities against AD-related enzymes. Notably, the extract acted as a BACE-1 blocker and reduced amyloid beta 42 (A $\beta 42$ ) peptides in Drosophila models, resulting in improved locomotor behaviors. Information gained from this study suggested that $D$. esculentum showed potential for $A D$ amelioration and prevention. Further investigations in vertebrates or humans are required to determine the effective doses of $D$. esculentum against AD, particularly via amyloidogenic pathway.

Alzheimer's disease (AD) is an irreversible, degenerative brain disease characterized by impairment of cognitive functions, accounting for $60-80 \%$ of all dementia patients ${ }^{1}$. Worldwide, over 43 million people live with dementia, and this number is expected to increase dramatically, rendering AD the fifth leading cause of death ${ }^{2}$. Neuropathological diagnosis of an AD brain reveals the loss of cholinergic neurons from the basal forebrain to the hippocampus, resulting in reduction of choline acetyltransferase (ChAT), an enzyme responsible for the synthesis of acetylcholine (ACh), a neurotransmitter that regulates the sleep cycle, memory and learning functions ${ }^{3}$. Reduction of ChAT activity and ACh levels are significantly corroborated with cognitive impairment in $\mathrm{AD}$ cases $^{4}$. Therefore, prevention of ACh deprivation by inhibiting acetylcholine-degrading enzymes, cholinesterases (acetylcholinesterase (AChE) and butyrylcholinesterase (BChE)) could be a therapeutic target for $\mathrm{AD}^{3}$. In addition to the cholinergic system, the accumulation of extracellular senile plaques consisting of deposits of amyloid beta $(\mathrm{A} \beta)$ peptides ultimately leads to neuronal cell death and brain atrophy ${ }^{5}$. Amyloid beta peptides are generated through successive cleavage of amyloid precursor proteins (APPs) by beta-secretase 1 (BACE-1) and gamma-secretase in brain tissues ${ }^{6,7}$. A $\beta$ peptides as either $A \beta 40$ or $A \beta 42$ are thought to be secreted and aggregated as senile plaques due to their low solubility ${ }^{8}$, with $A \beta 42$ the major and more neurotoxic component in amyloid plaques at the earliest stage of $\mathrm{AD}$. Patients with familial $\mathrm{AD}$ regularly exhibit a higher ratio of $\mathrm{A} \beta 42$ in the brain ${ }^{9}$. Cleavage of APPs by BACE- 1 is a rate-limiting step in A $\beta$ production; therefore, BACE- 1 inhibitors

${ }^{1}$ Institute of Nutrition, Mahidol University, Salaya, Phuttamonthon 73170, Nakhon Pathom, Thailand. ${ }^{2}$ Department of Biochemistry, Faculty of Medicine, Chiang Mai University, Meung, Chiang Mai 50200, Thailand. ${ }^{3}$ Department of Preclinical Science and Applied Animal Science, Faculty of Veterinary Science, Mahidol University, Salaya, Phuttamonthon 73170, Nakhon Pathom, Thailand. "Department of Pharmacology, Faculty of Medicine, Chiang Mai University, Meung, Chiang Mai 50200, Thailand. ${ }^{\circledR}$ email: piya.tem@mahidol.ac.th 


\begin{tabular}{|l|c|l|c|l|}
\hline \multirow{2}{*}{ Solvents } & & \multicolumn{3}{|l|}{ Antioxidant activities $(\boldsymbol{\mu m o l}$ TE/g dry weight) } \\
\cline { 3 - 5 } & TPCs $(\mathbf{m g ~ G A E} / \mathbf{g}$ dry weight) & DPPH radical scavenging assay & FRAP assay & ORAC assay \\
\hline Hexane & $3.58 \pm 0.25^{\mathrm{C}}$ & $0.06 \pm 0.30^{\mathrm{B}}$ & $12.88 \pm 0.29^{\mathrm{C}}$ & $162.42 \pm 6.15^{\mathrm{B}}$ \\
\hline Dichloromethane & $5.51 \pm 0.44^{\mathrm{B}}$ & $0.15 \pm 0.20^{\mathrm{B}}$ & $16.45 \pm 1.52^{\mathrm{B}}$ & $170.02 \pm 9.83^{\mathrm{B}}$ \\
\hline Ethanol & $21.58 \pm 0.91^{\mathrm{A}}$ & $1.82 \pm 0.54^{\mathrm{A}}$ & $192.11 \pm 10.31^{\mathrm{A}}$ & $645.91 \pm 8.74^{\mathrm{A}}$ \\
\hline
\end{tabular}

Table 1. Total phenolic contents (TPCs) and antioxidant activities of D. esculentum extracted with gradually increased polarity index solvents. Data are presented as mean \pm SD of three independent experiments. Capital letters within a column for a given parameter are significantly different at $p<0.05$. The final concentration of the extract was $1.25 \mathrm{mg} / \mathrm{mL}$.

are one of the attractive targets for $\mathrm{AD}$ drug development ${ }^{7}$. Several studies on $\mathrm{AD}$ onset and progression have been performed; however, currently, no effective treatments are available to prevent or ameliorate this debilitating neurodegenerative disease. Drug treatments for $\mathrm{AD}$ are primarily designed to slow down cognitive decline and ameliorate behavioral symptoms. These drugs often induce severe side effects including nausea, vomiting and liver damage ${ }^{10}$. Traditional medicines from natural products are currently of interest as promising alternative candidates for the treatment of $\mathrm{AD}$ because of their consumption safety, cost-effectiveness and limited side effects ${ }^{11}$.

Diplazium esculentum (Retz.) Sw., a common pteridophytes in the family Athyriaceae (also known as 'PakKood' in Thai), grows as an edible vegetable fern that is widely distributed in moist climatic areas. Only the young curly fronds are used as food ingredients in stir-fries and salads, and the fern is considered to have high economic value in Thailand. D. esculentum has been reported as a credible source of minerals and bioactive compounds such as phosphorus, potassium, alkaloids, flavonoids, saponins, tannins and terpenoids ${ }^{12-14}$ together with antibacterial, antidiabetic, antioxidant and hepatoprotective activities ${ }^{15,16}$. The methanolic crude extract of D. esculentum inhibited AChE activities in vitro, with half-maximal inhibitory concentration $\left(\mathrm{IC}_{50}\right)$ value at $272.97 \pm 19.38 \mu \mathrm{g} / \mathrm{mL}$, implying that it might be a promising candidate for prevention or treatment of AD by supporting cholinergic neurons ${ }^{17}$. However, it is unknown whether D. esculentum possesses anti-AD properties in vivo, particularly in relation to the amyloid pathway. Thus, here, the anti-AD properties of $D$. esculentum extract in vitro and in Drosophila models of AD were elucidated. Drosophila models have well-defined genetic characteristics including a short life span, simplicity in genetic manipulation, and a powerful binary UAS-GAL4 transgenic system, allowing tissue-specific protein expression ${ }^{18}$. AD-like symptoms can be obtained from flies expressing human $\mathrm{AD}$ genes that resemble $\mathrm{AD}$ pathology presented in mice AD models ${ }^{19}$. Flies are also a powerful alternative model for drug screening since their anatomy exhibits an open blood vascular system, hence drugs or plant extracts can be distributed to target organs, even the brain ${ }^{20}$. The blood-brain barrier (BBB) is known to restrict the distribution of biomolecules $(>500 \mathrm{kDa})$, including proteins and drugs ${ }^{21}$. Studies in the cell models cannot overcome this problem. Thus, here, phytochemical analysis, antioxidant activities, and in vitro anti-AD properties of D. esculentum extract through inhibition of the key enzymes relevant to $\mathrm{AD}$ (AChE, BChE and BACE-1) were studied. Additionally, inhibition of BACE- 1 and A $\beta 42$ production in Drosophila models were also elucidated.

\section{Results}

Total phenolic contents (TPCs) and antioxidant activities. To determine the optimal extraction method for D. esculentum, the sample was extracted with gradually increased polarity index solvents including hexane, dichloromethane and ethanol. Extraction yields (\%) were 10.1, 11.8 and 22.2, respectively, indicating that extraction yield increased with increasing solvent polarity. Ethanol is a suitable solvent for the extraction of various polar compounds. High recovery yield of the ethanolic extract resulted from the high concentration of polar bioactive compounds in D. esculentum. Likewise, lower recovery yields for dichloromethane and hexane extracts represented low contents of semi-polar and non-polar components, respectively. Bioactive compounds regarding TPCs and antioxidant activities were also tested. Table 1 shows that the ethanolic extract contained the highest TPCs compared to dichloromethane and hexane. Average TPC values of the ethanolic extract were approximately 5 times greater than dichloromethane and hexane. Average antioxidant activity of the ethanolic extract followed a similar pattern, with average DPPH radical scavenging value 12 and 30 times higher than dichloromethane and hexane, respectively. Average FRAP value of the ethanolic extract was 11 and 15 times higher than dichloromethane and hexane, respectively, while average ORAC value of the ethanolic extract was approximately 4 times higher than dichloromethane and hexane. Results implied that the sample with high TPCs extracted by ethanol also exhibited high antioxidant properties regarding DPPH radical scavenging, FRAP and ORAC assays.

In vitro anti-Alzheimer's disease properties. To investigate the anti-AD properties of D. esculentum extracted by different solvents in vitro, inhibitory enzyme assays toward AChE, BChE and BACE-1, as the major enzymes involved in $\mathrm{AD}$ pathogenesis, were employed. Results in Table 2 show that all D. esculentum extracts displayed anti-AChE, anti-BChE and anti-BACE-1 activities with varying degrees of inhibition. The ethanolic fraction exhibited the highest anti-AChE activity (46.15 $\pm 6.17 \%$ inhibition). Ethanol was the best solvent for obtaining bioactive compounds against AChE at approximately 2 to 5 times greater than dichloromethane and hexane. Conversely, Table 2 also revealed that anti-BChE activity of all D. esculentum extracts was more pro- 


\begin{tabular}{|l|l|l|l|}
\hline \multirow{2}{*}{ Solvents } & \multicolumn{2}{|l|}{ Enzyme inhibitory activities (\%) } & \multicolumn{2}{|l|}{ BACE-1 } \\
\cline { 2 - 4 } & AChE & BChE & $69.76 \pm 4.16^{\mathrm{A}}$ \\
\hline Hexane & $15.59 \pm 3.23^{\mathrm{C}}$ & $47.43 \pm 4.11^{\mathrm{A}}$ & $68.54 \pm 0.44^{\mathrm{A}}$ \\
\hline Dichloromethane & $24.19 \pm 3.92^{\mathrm{B}}$ & $49.85 \pm 4.29^{\mathrm{A}}$ & $55.91 \pm 5.32^{\mathrm{B}}$ \\
\hline Ethanol & $46.15 \pm 6.17^{\mathrm{A}}$ & $53.12 \pm 5.80^{\mathrm{A}}$ & \\
\hline
\end{tabular}

Table 2. Percentage enzyme inhibitory activities of $D$. esculentum extracted with hexane, dichloromethane and ethanol against AChE, BChE and BACE-1. Data are presented as mean \pm SD of three independent experiments. Capital letters within a column for a given parameter are significantly different at $p<0.05$. The final concentration of the extract was $1.25 \mathrm{mg} / \mathrm{mL}$.

\begin{tabular}{|l|l|l|}
\hline \multirow{2}{*}{} & Flavonoids $(\boldsymbol{\mu g} / \mathrm{g}$ extract) & Quercetin \\
\cline { 2 - 3 } & Rutin & $1,463.69 \pm 29.07$ \\
\hline Ethanolic extract of D. esculentum & $760.77 \pm 26.93$ & \\
\hline
\end{tabular}

Table 3. Quantification of rutin and quercetin in the ethanolic extract of D. esculentum.

nounced than anti-AChE activities. However, statistical analysis indicated that all fractions possessed the same inhibitory activities against BChE.

D. esculentum extracts were tested for their inhibitory properties against BACE-1, an enzyme involved in the amyloidogenic pathway. Inhibition of BACE-1 is currently one of the AD drug targets. All fractions appeared to inhibit BACE-1 with high efficacy (above $\mathrm{IC}_{50}$ value). Hexane and dichloromethane fractions exhibited antiBACE-1 activities significantly higher than the ethanolic fraction (Table 2). This result was interesting since the ethanolic fraction of D. esculentum constantly demonstrated high TPC, antioxidant activity, anti-AChE and antiBChE activities (Tables 1, 2). Bioactive compounds inhibiting BACE-1 may be different from those inhibiting AChE and BChE. Since hexane and dichloromethane extracts showed high anti-BACE-1 activities, the active compounds obstructing BACE-1 were probably hydrophobic, non-polar compounds.

Extraction yield and in vitro data regarding TPCs, antioxidant and enzyme inhibitory activities revealed that the ethanolic fraction of $D$. esculentum was the most potent among all the tested extracts (Tables 1,2). Thus, only the ethanolic fraction of $D$. esculentum was subjected to phenolic compound analysis utilizing high-performance liquid chromatography (HPLC) (Supplementary Figure S1 and S2). Only two flavonoids were identified, including quercetin $(1463.69 \pm 29.07 \mu \mathrm{g} / \mathrm{g}$ extract), and its glycosylated form, rutin $(760.77 \pm 26.93 \mu \mathrm{g} / \mathrm{g}$ extract) (Table 3).

Suppression of BACE-1 activities and $A \beta 42$ accumulation in flies co-expressing APP-BACE-1. The ethanolic extract of D. esculentum inhibited BACE-1 activity by approximately $50 \%$ in vitro (Table 2). Hence, the effect of $D$. esculentum extracts on BACE-1 activity in vivo using Drosophila was further determined. To represent human amyloidogenic pathway, flies carrying human amyloid precursor proteins (APPs) and human beta-secretase 1 (BACE-1) (APP-BACE-1) were utilized. Virgin females of the panneuronal elav-Gal4 driver were crossed with UAS-APP-BACE-1 males, resulting in F1 flies that co-expressed human APPs and BACE-1 (called AD flies). Moreover, elav-Gal4 flies were also included as AD-free flies. Deionized water (DI) was used as a chemical-free control, and $1 \%$ DMSO was used as a solvent control to dissolve donepezil and the extract. After eclosion, flies were treated with DI, 1\% DMSO, 125 and $250 \mu \mathrm{g} / \mathrm{mL}$ of $D$. esculentum as well as $10 \mu \mathrm{M}$ donepezil for 28 days. Afterward, head lysates were prepared without protease inhibition and subjected to BACE-1 assay. Figure 1A shows that both AD flies treated with DI or 1\% DMSO displayed the same levels of BACE-1 function, indicating that DI and 1\% DMSO did not affect BACE-1 activity in vivo, while $\mathrm{AD}$-free flies had no detectable BACE- 1 activity as expected. BACE-1 activity decreased by two folds in AD flies receiving $125 \mu \mathrm{g} / \mathrm{mL}$ of $D$. esculentum. This effect was considerably enhanced when AD flies received $250 \mu \mathrm{g} /$ $\mathrm{mL}$ of $D$. esculentum and $10 \mu \mathrm{M}$ donepezil. Donepezil was reported to inhibit BACE- 1 enzymes at an $\mathrm{IC}_{50}$ of $1.5 \mu \mathrm{M}^{22}$. Results suggested that the ethanolic fraction of D. esculentum ingested by flies inhibited BACE-1 activity in a dose-dependent manner.

Proteolytic cleavage of APPs by BACE-1 resulted in the formation of amyloid beta 42 (A $\beta 42$ ) peptides, a hallmark of $\mathrm{AD}^{23}$. Suppression of BACE-1 activity presented in AD flies raised the question whether the ethanolic extract of $D$. esculentum could prevent $\mathrm{A} \beta 42$ accumulation in $\mathrm{AD}$ flies. On day 28 of treatment, fly heads were collected and lysed with a lysis buffer containing protease and phosphatase inhibitors to prevent amyloid degradation. Equal amounts of protein supernatants were used to quantify human A $\beta 42$ using the ELISA method. In agreement with previous studies, Fig. $1 \mathrm{~B}$ shows that $\mathrm{AD}$ flies treated with $\mathrm{DI}$ or $1 \% \mathrm{DMSO}$ accumulated high $\mathrm{A} \beta 42$ at similar levels, while $\mathrm{A} \beta 42$ was not detected in elav-Gal4 (AD-free flies, data not shown). Results indicated that the fly model was representative of human amyloidogenic pathway. AD flies that received $250 \mu \mathrm{g} / \mathrm{mL}$ of $D$. esculentum as well as $10 \mu \mathrm{M}$ donepezil exhibited significantly decreased levels of A $\beta 42$ compared to DI and $1 \%$ DMSO. D. esculentum and donepezil influenced BACE-1 activity (Fig. 1B). However, this was not potent enough to reduce $\mathrm{A} \beta 42$ deposits, although a low concentration of D. esculentum at $125 \mu \mathrm{g} / \mathrm{mL}$ reduced BACE- 1 activity by approximately $50 \%$ in the tested flies. The ethanolic fraction of $D$. esculentum may prevent AD by reducing A 342 deposits via BACE- 1 inhibition in a dose-dependent manner. 
A.

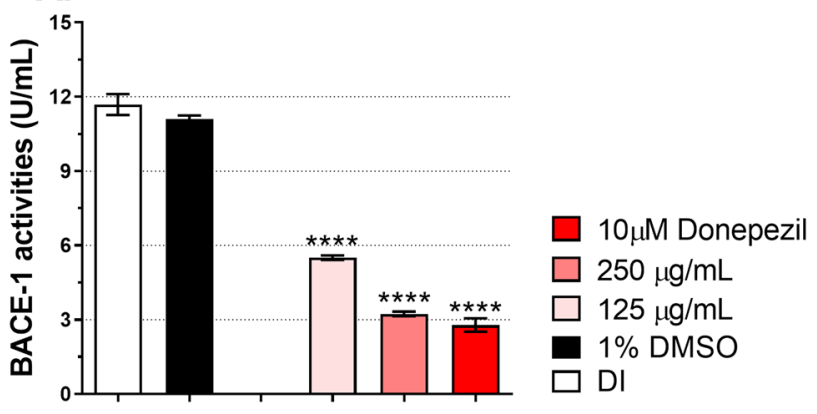

B.

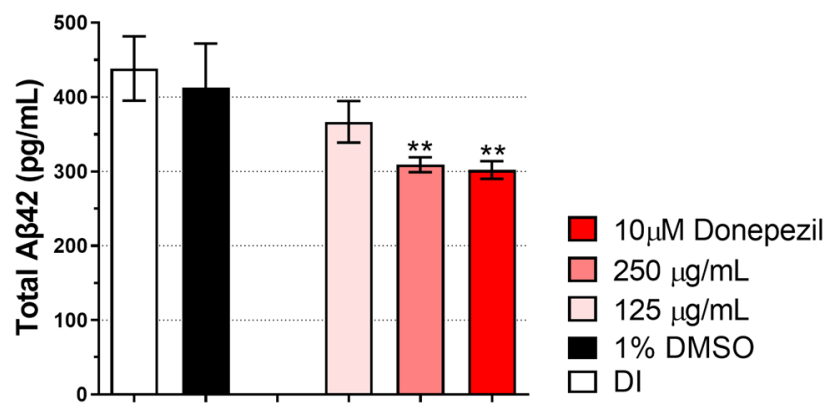

Figure 1. Effect of the ethanolic extract of D. esculentum on (A) BACE-1 activities and (B) A $\beta 42$ accumulation in the Drosophila models of AD. AD-free flies (elav-Gal4) were cultured on a normal medium, and AD flies (APP-BACE-1) were cultured on a medium containing deionized water (DI), $1 \%$ DMSO, $125 \mu \mathrm{g} / \mathrm{mL}$ and $250 \mu \mathrm{g} / \mathrm{mL}$ of D. esculentum and $10 \mu \mathrm{M}$ donepezil for 28 days before assay for either BACE- 1 activities or A $\beta 42$ levels. Values are mean \pm SD of three assays and statistical significance was analyzed against $\mathrm{AD}$ flies (DI) by one-way ANOVA followed by Tukey's test. $\left.{ }^{* *}, p<0.01\right)$ and ${ }^{* * *}, p<0.0001$.

A.

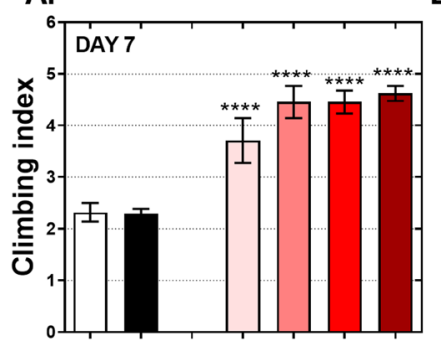

B.

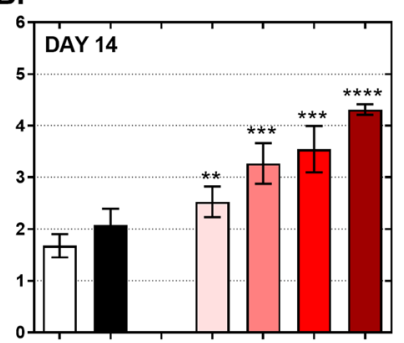

C.

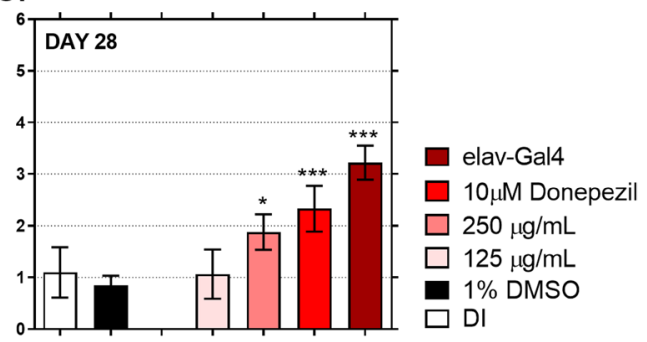

Figure 2. Effects of the ethanolic extract of D. esculentum on Drosophila climbing ability. AD-free flies (elavGal4) cultured on a normal medium, and AD flies (APP-BACE-1) cultured on a medium containing deionized water (DI), $1 \%$ DMSO, $125 \mu \mathrm{g} / \mathrm{mL}$ and $250 \mu \mathrm{g} / \mathrm{mL}$ of D. esculentum and $10 \mu \mathrm{M}$ donepezil for the indicated days were assessed for climbing ability. Values are mean \pm SD of three assays and statistical significance was analyzed against $\mathrm{AD}$ flies (DI) by one-way ANOVA followed by Tukey's test. ${ }^{*}, p<0.05$; ${ }^{* *}, p<0.01$; $^{* * *}, p<0.001$ and ${ }^{* * *}$, $p<0.0001$.

Climbing behavior in flies co-expressing APP-BACE-1. Results in Fig. 1A and B showed that the ethanolic extract of D. esculentum inhibited BACE-1 activity in flies expressing APP and BACE-1, resulting in reduced production of $\mathrm{A} \beta 42$ in fly brains. Uniquely, most neurodegenerative diseases, including $\mathrm{AD}$, can be characterized by age-dependent deterioration in climbing behavior in Drosophila. Thus, reduction in A $\beta 42$ accumulation leading to improvement of Drosophila climbing behavior was examined. As illustrated in Fig. 2A, the $\mathrm{AD}$-free flies (elav-Gal4) climbed at a climbing index of 4.5 on day 7 after eclosion. The climbing index gradually reduced with increased fly age (Fig. 2B,C), supporting the age-dependent phenotype. In AD flies, both those with DI or $1 \%$ DMSO showed severe climbing index as early as day 7 . This defect increased with aging, confirming that $\mathrm{AD}$ caused severe damage to the fly brain, leading to poor climbing ability. Interestingly, flies with two different concentrations of D. esculentum $(125 \mu \mathrm{g} / \mathrm{mL}$ and $250 \mu \mathrm{g} / \mathrm{mL})$ and $10 \mu \mathrm{M}$ donepezil showed reduced climbing defects. On day 7 after eclosion, compared to $\mathrm{AD}$ and $\mathrm{AD}$-free control flies, flies receiving $250 \mu \mathrm{g} / \mathrm{mL}$ of D. esculentum and $10 \mu \mathrm{M}$ donepezil could fly as high as the AD-free flies, while flies that received $125 \mu \mathrm{g} /$ 
A.

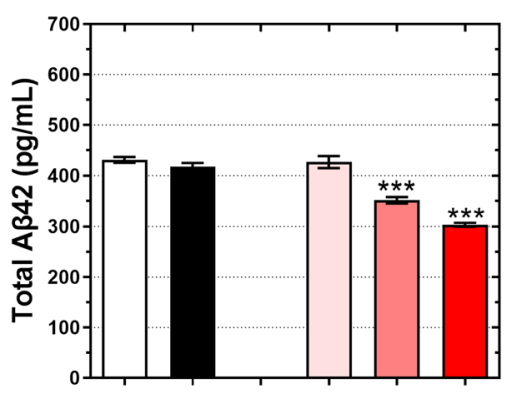

B.

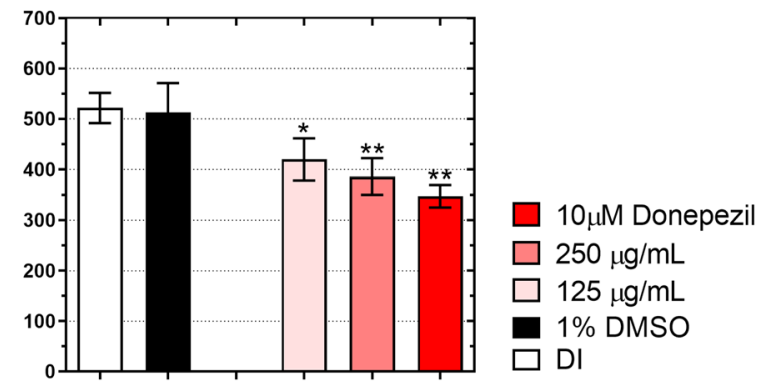

Figure 3. Effect of the ethanolic extract of D. esculentum on A $\beta 42$ accumulation in Drosophila models as (A) flies expressing A $\beta 42$ (chr.2) and (B) flies expressing A $\beta 42$ (chr.3). AD-free flies (elav-Gal4) were cultured on a normal medium, while $\mathrm{AD}$ flies $(\mathrm{A} \beta 42)$ were cultured on a medium containing deionized water (DI), $1 \%$ DMSO, $125 \mu \mathrm{g} / \mathrm{mL}$ and $250 \mu \mathrm{g} / \mathrm{mL}$ of $D$. esculentum and $10 \mu \mathrm{M}$ donepezil for 28 days and then assayed for A $\beta 42$ levels. Values are mean \pm SD of three assays and statistical significance was analyzed against AD flies (DI) by one-way ANOVA followed by Tukey's test. ${ }^{*}, p<0.05$; $^{* *}, p<0.01 ;{ }^{* *}, p<0.001$.

$\mathrm{mL}$ of D. esculentum showed moderate rescuing ability. Although flies that received $250 \mu \mathrm{g} / \mathrm{mL}$ of D. esculentum climbed progressively slower as they aged, the climbing index was statistically similar to flies with $10 \mu \mathrm{M}$ donepezil at all times tested, suggesting the rescuing activity of $D$. esculentum at $250 \mu \mathrm{g} / \mathrm{mL}$ in climbing behavior. Unfortunately, D. esculentum at $125 \mu \mathrm{g} / \mathrm{mL}$ delayed the climbing index by up to 14 days; therefore, this dose of ethanolic extract of $D$. esculentum should not be used, even though it can suppress A $\beta 42$ accumulation (Fig. 1B).

A $\beta 42$ accumulation and climbing behavior in flies expressing $A \beta 42$. Figure 1 shows that $D$. esculentum extract reduced $\mathrm{A} \beta 42$ quantity by influencing BACE-1 activities. To determine whether $D$. esculentum reduced $A \beta 42$ deposit, two strains of flies carrying $A \beta 42$ comprising of $A \beta 42$ chr.2 and $A \beta 42$ chr. 3 were investigated. Virgin females of elav-Gal4 were crossed with either UAS-A $\beta 42$ chr. 2 or UAS-A $\beta 42$ chr. 3 males, resulting in F1 flies that expressed human A $\beta 42$ peptides (called AD flies). Elav-Gal4 flies were also included as ADfree flies. After eclosion, the AD flies were subsequently treated with DI, $1 \%$ DMSO, 125 and $250 \mu \mathrm{g} / \mathrm{mL}$ of $D$. esculentum as well as $10 \mu \mathrm{M}$ donepezil. Flies were treated for 28 days. On day 28, fly heads were collected and quantified for human $\mathrm{A} \beta 42$, as previously described. Figure $3 \mathrm{~A}$ and $\mathrm{B}$ show that $\mathrm{AD}$ flies treated with $\mathrm{DI}$ or $1 \%$ DMSO had high levels of A $\beta 42$ peptide, whereas elav-Gal4 did not express A $\beta 42$ (data not shown). AD flies that received $250 \mu \mathrm{g} / \mathrm{mL}$ of $D$. esculentum as well as $10 \mu \mathrm{M}$ donepezil showed significantly decreasing $A \beta 42$ peptides compared to DI and 1\% DMSO. However, D. esculentum at $125 \mu \mathrm{g} / \mathrm{mL}$ did not prevent A $\beta 42$ deposit when A $\beta 42$ chr.2 flies were used. By contrast, D. esculentum at $125 \mu \mathrm{g} / \mathrm{mL}$ exhibited considerable A $\beta 42$ suppression when A $\beta 42 \mathrm{chr} .3$ flies were used (Fig. 3B). It was not possible to explain this dissimilarity between A $\beta 42 \mathrm{chr} .2$ and A $\beta 42$ chr.3; however, D. esculentum showed neuroprotective properties by suppression of A $\beta 42$ deposit.

As shown in Fig. 3, the ethanolic extract of $D$. esculentum reduced A $\beta 42$ accumulation in both strains of flies expressing $A \beta 42$. D. esculentum reduced $A \beta 42$ aggregation while enhancing $A \beta 42$ clearance from fly brains. Reduction in A $\beta 42$ accumulation and whether it led to improvement of Drosophila behaviors was tested. As demonstrated in Fig. 4, AD-free flies (elav-Gal4) had a climbing index of 5 on day 7 after eclosion. The climbing index declined slowly on day 14 and day 28 (Fig. 4). AD flies (A $\beta 42 \mathrm{chr} .2$ ) fed with DI or 1\% DMSO as control were approximately two-folds slower compared to $\mathrm{AD}$-free flies, demonstrating severe climbing deficits as early as day 7. Also, this defect continuously influenced climbing ability at day 14 and day 28, confirming that A $\beta 42$ peptides caused severe neurotoxicity, eventually leading to poor climbing ability (Fig. 4A-C). During treatment, flies with two different concentrations of D. esculentum $(125 \mu \mathrm{g} / \mathrm{mL}$ and $250 \mu \mathrm{g} / \mathrm{mL})$ and $10 \mu \mathrm{M}$ donepezil showed significant climbing improvement compared to AD flies. However, flies that received $125 \mu \mathrm{g} / \mathrm{mL}$ of $D$. esculentum showed moderate rescuing ability that lasted for 14 days. AD flies treated with $250 \mu \mathrm{g} / \mathrm{mL}$ of $D$. esculentum exhibited comparable rescuing ability with flies treated with $10 \mu \mathrm{M}$ donepezil, albeit to a lesser extent. AD flies (A $\beta 42$ chr.3) were used and tested following the same procedure as A $\beta 42$ chr.2 (Fig. 4D-F). Results concurred when $\mathrm{A} \beta 42 \mathrm{chr} .2$ was used, indicating that different locations of gene modification did not interfere with AD pathogenesis in the AD flies. Importantly, this suggested that the ethanolic fraction of D. esculentum at $250 \mu \mathrm{g} /$ $\mathrm{mL}$ could be used as a therapeutic dose in AD flies when taken together with the obtained data.

\section{Discussion}

Alzheimer's disease $(\mathrm{AD})$ is an irreversible, degenerative brain disease leading to dementia. Regrettably, current medicines show side effects, low efficacy, and cannot rescue AD pathogenesis. AD complexities are associated with several pathways involving oxidative stress, tau hyperphosphorylation, inflammation, cholinesterases, and BACE-1 functions; thus, safe and multi-targeted medicine could be ideal for AD drugs. Vegetables and plant extracts are currently under-investigated for their health-promoting activities, including anti-AD properties. Several pieces of evidence illustrate the potential anti-AD properties of phytochemicals, including phenolic acids and flavonoids both in vitro and in vivo ${ }^{24}$. Here, results elucidated the anti-AD properties of an edible fern with high economic value as D. esculentum in vitro and using Drosophila models of AD. The main findings were (1) ethanol 
A.

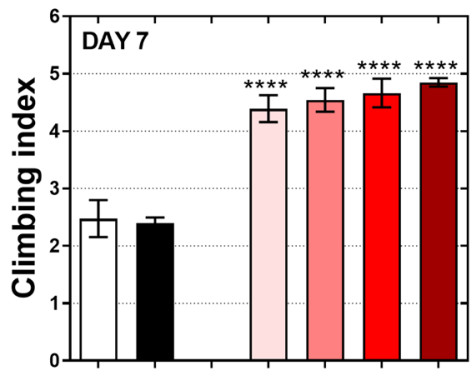

B.

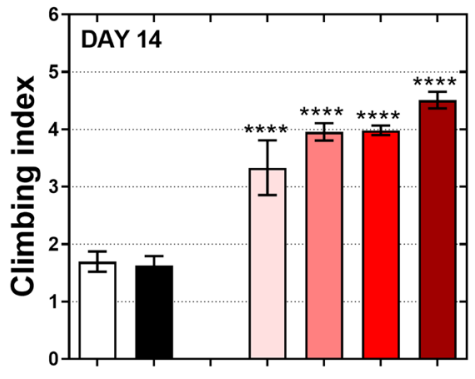

C.

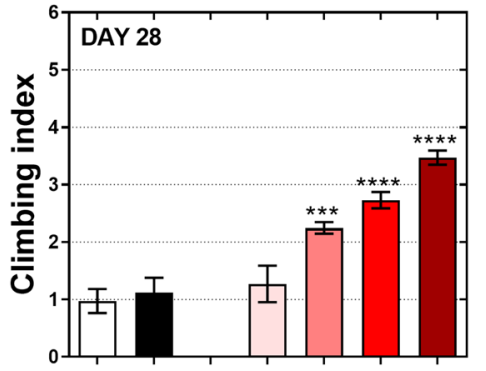

D.

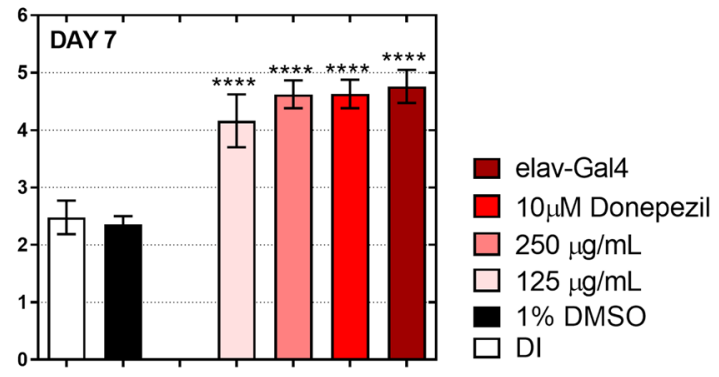

E.

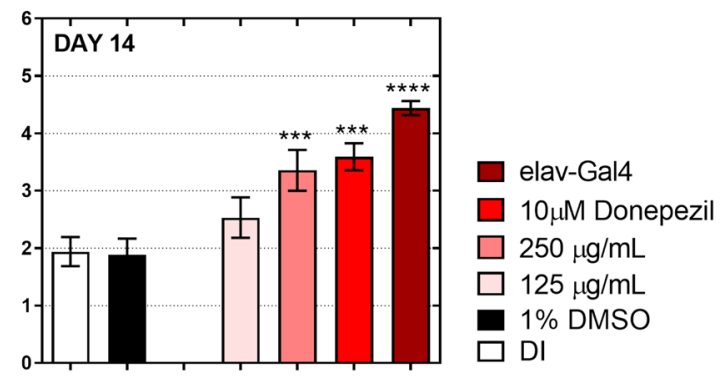

F.

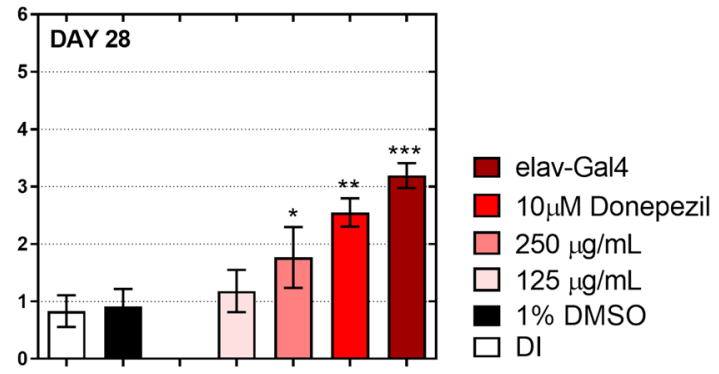

Figure 4. Effect of the ethanolic extract of D. esculentum on climbing ability of flies expressing A $\beta 42,(\mathbf{A}-\mathbf{C})$ flies expressing A $\beta 42$ (chr.2), and (D-F) flies expressing A $\beta 42$ (chr.3). AD-free flies (elav-Gal4) were cultured on a normal medium, while $\mathrm{AD}$ flies $(\mathrm{A} \beta 42)$ were cultured on a medium containing deionized water (DI), $1 \% \mathrm{DMSO}, 125 \mu \mathrm{g} / \mathrm{mL}$ and $250 \mu \mathrm{g} / \mathrm{mL}$ of $D$. esculentum and $10 \mu \mathrm{M}$ donepezil for the indicated days and then assayed for climbing ability. Values are mean \pm SD of three assays and statistical significance was analyzed against AD flies (DI) by one-way ANOVA followed by Tukey's test. ${ }^{*}, p<0.05 ;{ }^{* *}, p<0.01$; $^{* * *}, p<0.001$.

was an appropriate solvent for extracting anti-AD compounds from D. esculentum, (2) ethanolic extracts were rich in phenolic compounds, particularly rutin and its aglycone, quercetin, that might contribute to antioxidant and inhibitory activities against AD-related enzymes, and (3) the ethanolic extract inhibited BACE-1 activities, a rate-limiting step in amyloid beta production, and also directly interfered with A $\beta 42$ levels in Drosophila brain.

Based on the high antioxidant activities detected in hydrophilic solvents (ethanol), the antioxidative agents presented in D. esculentum might be flavonoids and phenolic acids that dissolve better in hydrophilic solvents compared to hexane and dichloromethane. Ethanol is a versatile solvent that is preferably used to dissolve polar substances with some contamination of non-polar compounds. Phenolics such as flavonoids are plant secondary metabolites reported for their health benefits, including their high antioxidant properties ${ }^{25}$. Results indicated that the ethanolic fraction from D. esculentum possessed high TPCs and antioxidant activities and showed correlation between the amount of TPCs and antioxidant properties (Table 1). This finding was consistent with a previous study demonstrating that the methanolic fraction of $D$. esculentum had high antioxidant properties compared to the chloroform fraction ${ }^{26}$. An HPLC analysis of the ethanolic fraction of D. esculentum found only rutin and quercetin. Interestingly, an earlier report showed that $D$. esculentum is rich in procyanidin, quercetin3-rutinoside (rutin), kaempferol-3-rutinoside, quercetin-3-glucoside, and eriodictyol 5-O-methyl ether 7- $\beta$-Dxylosylgalactoside, which are flavonoids ${ }^{27}$. Flavonoid structures mainly consist of hydroxyl groups, 2-3 double bonds, and 4-oxo functions, promoting antioxidant activities ${ }^{28}$.

Results revealed that the ethanolic fraction exhibited high enzyme inhibitory activities toward AChE, BChE and BACE-1 covering both cholinergic and amyloid hypotheses at 46, 53 and 56\%, respectively (Table 2). Findings implied that the AChE, BChE and BACE-1 inhibitors present in D. esculentum might be polar compounds, in parallel with antioxidant data. Two flavonoids detected in D. esculentum extract, rutin and quercetin, might be 
responsible for these inhibitory activities. However, it has been documented that rutin and quercetin inhibited AChE reaction with half maximal inhibitory concentration $\left(\mathrm{IC}_{50}\right)$ of 219 and $181 \mu \mathrm{M}$, respectively, while these two flavonoids also inhibited BChE reaction with $\mathrm{IC}_{50}$ values of 288 and $203 \mu \mathrm{M}$, respectively ${ }^{29}$. Nevertheless, the concentration of rutin and quercetin presented in the D. esculentum extract during enzyme assays were 1.56 and $6.05 \mu \mathrm{M}$, respectively, implying that rutin and quercetin may synergist with other unknown active compound(s) in the extract to inhibit $\mathrm{AChE}$ and $\mathrm{BChE}$ activities, which should be further explored. Interestingly, quercetin effectively inhibited BACE- 1 reaction with $\mathrm{IC}_{50}$ values of $5.4 \mu \mathrm{M}^{30}$. In addition, among 448 clinical compounds (NIH clinical collection), rutin was identified as a potential BACE-1 inhibitor (effective at a dose of $1 \mu \mathrm{M}$ ) by a systematic approach called A $\beta$ PP-selective BACE inhibitors (ASBI) ${ }^{31}$. Thus, due to their low $\mathrm{IC}_{50}$ values against BACE-1, these flavonoids may act as strong inhibitors against BACE-1, leading to high enzyme inhibition detected in the assay containing D. esculentum ethanolic extract (Table 2).

We molecularly explored our finding in-depth in vivo using Drosophila models. Over the last decade, an increasing number of studies have used Drosophila as a model to study AD. Our model was developed based on Wang et al. ${ }^{32}$, who assessed the anti-AD effect of curcuminoids in flies co-expressing human APPs and BACE-1. Their data revealed that curcuminoids could rescue rough eye phenotypes, and climbing behaviors via BACE-1 inhibitory activities, confirming the appropriate use of Drosophila as a model for studying plant extracts on amyloidogenic pathway. Two hypotheses were tested. The first was the effect of D. esculentum extract on Drosophila co-expressing human APPs and BACE-1 that represent human amyloid cascades. The second was the direct effect of the extract on Drosophila expressing human A $\beta 42$ peptides. This model was assessed to determine the direct role of $D$. esculentum on prevention of $A \beta 42$ toxicity. We found that the ethanolic extract acted as a BACE- 1 blocker and also directly reduced $A \beta 42$ peptides in vivo (Figs. 1, 2, 3 and 4). Although a lower concentration of $D$. esculentum might be used in the early stage of $\mathrm{AD}$, a higher concentration is recommended for progressive deterioration of AD. The D. esculentum extract inhibited BACE-1 activity in fly brains. This infers that the extract can be diffused across the Drosophila blood-brain barrier (BBB). One of the major obstacles in AD drug development is the inability to traverse the BBB. A compound that can pass the BBB should comprise lipophilic molecules with molecular weight less than $400-500 \mathrm{kDa}^{21}$. Phenolic compounds may be suitable as AD drug candidates due to their small molecular weight $(<400 \mathrm{Da})$. There are two mechanisms underlying inhibition of BACE-1 as (1) direct interaction between compounds and enzymes, and (2) interference of BACE-1 expression. The first was demonstrated by Shimmyo et al. They found that quercetin inhibited BACE-1 activities with $\mathrm{IC}_{50}$ values of $5.4 \mu \mathrm{M}$ via direct inhibition at the enzyme active site ${ }^{30}$. For the second mechanism, Huang et al. showed that BACE-1 expression could be suppressed via estrogen receptor $\beta$ (ER $\beta$ ) and nuclear factor-kappa $B$ $(\mathrm{NF \kappa B})$ signaling by curcumin, a polyphenol from turmeric ${ }^{33}$. The orthologs of these proteins can also be found in Drosophila ${ }^{34,35}$. Thus, D. esculentum may directly inhibit BACE-1 activities, BACE-1 expression and eventually $A \beta 42$ formation. We predict that quercetin, rather than rutin, was an active component in D. esculentum extract because rutin will be hydrolyzed to quercetin in the digestive system, allowing quercetin to be absorbed ${ }^{36}$.

How D. esculentum directly reduces A $\beta 42$ peptides in Drosophila remains unclear (Fig. 4). This study found that $D$. esculentum contains phenolic compounds such as quercetin (Table 3 ). These compounds might directly prevent $A \beta 40$ and $A \beta 42$ formation and extension ${ }^{37}$. Quercetin has been reported to reduce $A \beta 42$ toxicity in Drosophila-expressing $A \beta 42$ peptides via restoring gene expression disturbed by $A \beta$ toxicity, including cyclin $\mathrm{B}^{38}$. Furthermore, it has been reported that cholinesterases (AChE and $\mathrm{BChE}$ ) are associated with aggregation of $A \beta$ peptides and acetylcholinesterase inhibitor (donepezil) reduced acetylcholinesterase-mediated $A \beta$ aggregation $^{39}$. Thus, it could be possible that donepezil and the D. esculentum extract, which exhibited AChE and BChE inhibitory activities (Table 2) reduced A $\beta 42$ accumulation in flies expressing $A \beta 42$ via inhibition of $\mathrm{AChE}$ and BChE. Accumulation of amyloid peptides in nerve cells formed senile plaques and generated reactive oxygen species (ROS) ${ }^{40}$. ROS are derived from degenerating neurons. Our results showed that D. esculentum also contains unidentified phenolic acids and flavonoids that might contribute to high antioxidant activities, particularly through hydrogen atom transfer (HAT), as indicated by the ORAC data (Table 1$)^{41}$. Hence, the role of antioxidant activity of $D$. esculentum cannot be excluded.

Although, in the present study, there is no evidence that the decrease in $A \beta$ is a direct cause of the increase in climbing ability, several articles have shown that amyloid peptide expression in the Drosophila lead to (1) apoptotic cell death in the fly brain ${ }^{42},(2)$ defect in fly neuroanatomy ${ }^{43},(3)$ amyloid peptide deposit and aggregate in fly central nervous system $(\mathrm{CNS})^{43-45}$, (4) cell body and neuropil degeneration ${ }^{45}$ and (5) reduced glial cell number in $A \beta$-expressing brains ${ }^{46}$. All defects (1-5) lead to poor climbing ability. Hence, our flies which expressed $\mathrm{A} \beta$ could also mimic the same finding. However, further experiments, including apoptosis in fly brain tissues, neurodegeneration in the fly brain, $A \beta$-mediated ROS levels and degree of $A \beta$ aggregation should be considered.

\section{Material and methods}

Sample preparation and extraction. Diplazium esculentum (Retz.) Sw. (D. esculentum) was collected from Chiang Mai, Thailand. The sample collection was conducted following the guidelines and regulations of the legislation and the sample was identified by Dr. Kanchana Pruesapan (taxonomist), Plant Varieties Protection Division, Department of Agriculture, Bangkok. The plant sample was deposited in Bangkok Herbarium (BK), Bangkok, Thailand. The herbarium voucher specimen is BK069943. Edible part of D. esculentum (young leaves and young stems) was washed with deionized water and cut into small pieces. The sample was freeze-dried (Heto PowerDry PL9000, Heto Lab Equipment, Allerød, Denmark) and then ground into fine powder. Moisture content of the powder was determined using a Halogen moisture analyzer (HE53 series, MettlerToledo AG, Greifensee, Switzerland). The dry powder was extracted with hexane or dichloromethane or ethanol at $30^{\circ} \mathrm{C}$ for $2 \mathrm{~h}$. The mixture was then centrifuged at $3000 \mathrm{~g}$ for $20 \mathrm{~min}$ and the supernatant was subsequently 
collected. The solvent was removed using a rotary evaporator (model Eyela N-1200 Series). The dry extract was weighed, redissolved in DMSO, and kept at $-20^{\circ} \mathrm{C}$ until required for further analysis.

Phytochemical analysis. Total phenolic contents (TPCs) were determined according to the Folin-Ciocalteu method adapted from Ainsworth and Gillespie ${ }^{47,48}$. The TPCs were measured at a wavelength of $765 \mathrm{~nm}$ using a microplate reader. Gallic acid $(10,20,40,60,80,100$ and $200 \mu \mathrm{g} / \mathrm{mL})$ was used as the standard, and TPCs were expressed in gallic acid equivalents (GAE) per $1 \mathrm{~g}$ dry weight of sample.

The ethanolic fraction of $D$. esculentum was investigated for phenolic compounds using high-performance liquid chromatography (HPLC) (UtiMate HPLC with a HPG-3400SD pump equipped with photodiode array detector from Dionex, Sunnyvale, CA, USA) and C18 column $(250 \times 4.6 \mathrm{~mm}, 5 \mu \mathrm{m})$ (Agilent Technologies, Santa Clara, CA, USA), following sample preparation and HPLC conditions previously described ${ }^{49}$. The chromatogram was compared to phenolic standards, including apigenin, caffeic acid, catechin, chlorogenic acid, ferulic acid, gallic acid, hesperitin, isorhamnetin, kaempferol, luteolin, myricetin, naringenin, quercetin, rosmarinic acid and vanillic acid. In addition, $10 \mathrm{mg} / \mathrm{mL}$ of extract was diluted in $1 \mathrm{~mL}$ of methanol and injected onto the column, followed by the aforementioned HPLC condition, to measure the glycosylated form of quercetin, quercetin3-rutinoside or rutin.

Antioxidant activities. The established antioxidant assays including 2,2-diphenyl-1-picrylhydrazyl (DPPH) scavenging activity, ferric ion reducing antioxidant power (FRAP), and oxygen radical absorbance capacity (ORAC) $)^{50-53}$ were employed to evaluate the antioxidant activities of hexane, dichloromethane and ethanolic fraction of D. esculentum.

Inhibitory activities against AD-related enzymes. AChE inhibitory activities were assayed as formerly detailed by Jung et al. 2009 and Temviriyanukul et al. $2020^{53-55}$. In brief, $20 \mathrm{ng}$ of Electrophorus electricus AChE (1,000 units/mg, $100 \mu \mathrm{L}$ ) in $50 \mathrm{mM} \mathrm{KPB} \mathrm{(pH} \mathrm{7.0)} \mathrm{was} \mathrm{mixed} \mathrm{with} 16 \mathrm{mM}$ 5,5-dithio-bis-(2-nitrobenzoic acid) (DTNB, $10 \mu \mathrm{L}), 0.8 \mathrm{mM}$ acetylthiocholine $(40 \mu \mathrm{L})$ in $50 \mathrm{mM} \mathrm{KPB}(\mathrm{pH} 7.0)$, and D. esculentum extract (50 $\mu \mathrm{L}$ ). The reaction was measured using a microplate reader (Synergy ${ }^{\text {Tx }}$ HT 96-well UV-visible spectrophotometer with Gen5 data analysis software from BioTek Instruments, Inc., Winooski, VT, USA) at $412 \mathrm{~nm}$. Inhibitory percentage was calculated as follows:

$$
\% \text { inhibition }=\left(1-\frac{B-b}{A-a}\right) \times 100,
$$

where $A$ is the initial velocity of the reaction with enzyme, $a$ is the initial velocity of the reaction without enzyme, $B$ is the initial velocity of the enzyme reaction with extract, and $b$ is the initial velocity of the reaction with extract but without enzyme.

The BChE inhibitory activities of D. esculentum extract were tested as AChE assay. A total of $100 \mathrm{ng}$ equine serum BChE ( $\geq 10$ units/mg protein, $100 \mu \mathrm{L}$ ) in $50 \mathrm{mM} \mathrm{KPB}(\mathrm{pH} 7.0)$ containing $1 \mathrm{mM} \mathrm{MgCl}_{2}$ and $0.1 \mathrm{mM}$ butyrylthiocholine (BTCh) in $50 \mathrm{mM} \mathrm{KPB} \mathrm{(pH} \mathrm{7.0)} \mathrm{were} \mathrm{used} \mathrm{as} \mathrm{the} \mathrm{reaction} \mathrm{enzyme} \mathrm{and} \mathrm{substrate,}$ respectively ${ }^{54,55}$.

Fluorescence resonance energy transfer (FRET) on a BACE-1 activity detection kit (Sigma-Aldrich, MO, USA) was used to determine BACE-1 activity following the manufacturer's instructions.

Drosophila stocks and ethical approval. The elav-GAL4 (w[*]; P $\{\mathrm{w}[+\mathrm{mC}]=$ GAL4-elav.L $\}$ CG16779[3]) (BDSM 8760), UAS-APP-BACE-1 (w[1118]; P $\{\mathrm{w}[+\mathrm{mC}]=\mathrm{UAS}-\mathrm{APP} 695-\mathrm{N}-\mathrm{myc}\} \mathrm{TW6}, \mathrm{P}\{\mathrm{w}[+\mathrm{mC}]=\mathrm{UAS}-$ BACE1.Exel $\} 1 b)$ (BDSM 33798), UAS-A 342 (chr.2) (w[1118]; $\mathrm{P}\{\mathrm{w}[+\mathrm{mC}]=$ UAS-APP.Abeta42.B $\} \mathrm{m} 26 \mathrm{a})$ (BDSM $33769)$ and UAS-A $\beta 42(\mathrm{chr} .3)\left(\mathrm{w}{ }^{*}\right] ; \mathrm{P}\{\mathrm{w}[+\mathrm{mC}]=$ UAS-Abetal-42.G $\}$ ) (BDSM 32037) were obtained from Bloomington Drosophila stock center (Bloomington, IN, USA). All fly stocks were maintained in a standard medium at $25^{\circ} \mathrm{C}$. Based on the UAS/GAL4 system, crossing between an elav-GAL4 (driver) and a UAS strain resulted in F1 progenies expressing the desired expression of a specific protein in the fly brain. Schematic diagrams of Drosophila crosses and studies are illustrated in supplementary Figure S3. The Drosophila studies were approved by Mahidol University Institute of Animal Care and Use Committee (MU-IACUC) (COA.No.MUIACUC 2017/024) and the Institute of Nutrition, Mahidol University Institutional Animal Care and Use Committee (INMU-IACUC) (COA.No.MU-IACUC 2021/01).

Drosophila treatment. The newly eclosed F1 progenies (1-2 days old) were fed with instant Drosophila medium blue (Carolina Biological Supply Company, Burlington, North Carolina, USA) containing safety doses of D. esculentum ethanolic fraction $(125$ and $250 \mu \mathrm{g} / \mathrm{mL}), 1 \%$ DMSO or $10 \mu \mathrm{M}$ donepezil (cholinesterase and BACE-1 inhibitor ${ }^{22}$ ) and cultured at $28^{\circ} \mathrm{C}$ for the indicated time.

Climbing assay. To determine the neurodegenerative defect underlining locomotory coordination, the climbing or negative geotaxis assay was used ${ }^{56}$. In brief, on the indicated day after treatment, flies were transferred to a clean vial without anesthesia and given $15 \mathrm{~min}$ for acclimatization at $25^{\circ} \mathrm{C}$. The climbing index of each tested experiment was determined as follows: the number of flies in each score multiplied by the score they reached, and then divided by the total number of flies in each group. 
Determination of A $\beta 42$ Levels and BACE-1 activities in fly brain lysate. For A $\beta 42$ quantification, twenty-five to thirty fly heads were separated and collected using centrifugation and then mechanically homogenized in $100 \mu \mathrm{L}$ of $5 \mathrm{M}$ guanidine- $\mathrm{HCl}$ containing Protease Inhibitor Cocktail (Thermo Scientific ${ }^{\mathrm{m}}$ ). The samples were then centrifuged at $12,000 \mathrm{~g}$ for $15 \mathrm{~min}$ at $4{ }^{\circ} \mathrm{C}^{57,58}$. Protein supernatants were determined for protein concentration using Pierce BCA Protein Assay Kit (Bicinchoninic acid assay, Thermo Scientific ${ }^{\mathrm{rm}}$ ). Before sample loading, serial dilution of supernatants was performed using ELISA diluent buffer containing Protease Inhibitor Cocktail following the manufacturer's instructions (Life Technologies, Invitrogen). The samples were then measured at $450 \mathrm{~nm}$. Concentration of A $\beta 42$ peptides was calculated and compared with the standard control (recombinant human $\mathrm{A} \beta 42$ ). Data are presented as mean $\pm \mathrm{SD}$ of three experiments.

For BACE-1 activities, twenty-five to thirty fly heads were separated and collected using centrifugation and then mechanically homogenized in $100 \mu \mathrm{L}$ of T-PER ${ }^{\mathrm{mm}}$ Tissue Protein Extraction Reagent. Then, $2 \mu \mathrm{L}$ of protein samples (conc. $3 \mathrm{mg} / \mathrm{mL}$ ) were used to measure BACE-1 activity in the brain lysate. All reactions were performed in a 96-well microplate using a BACE-1 activity detection kit (Sigma-Aldrich, MO, USA). Results were presented as units $/ \mathrm{ml}(\mathrm{U} / \mathrm{mL})$. One unit of BACE-1 activity means it hydrolyzes 1.0 pmol of 7-methoxycoumarin-4-acetyl[Asn670, Leu671]-amyloid $\beta / A 4$ precursor protein 770 fragment 667-676-(2,4-dinitrophenyl)Lys-Arg-Arg amide substrate per minute at $\mathrm{pH} 4.5,37^{\circ} \mathrm{C}$. Data are presented as mean $\pm \mathrm{SD}$ of three experiments.

\section{Conclusion}

Results showed that an ethanolic extract of D. esculentum contained high phenolics, especially quercetin and kaempferol, that might contribute to antioxidant and anti-enzyme activities associated with $\mathrm{AD}$ pathogenesis in vitro. Moreover, the extract significantly decreased BACE- 1 activities and A $\beta 42$ peptide accumulation, while improving locomotor functions in Drosophila flies carrying human APPs and human BACE-1 or human A $\beta 42$ in their brains. Information obtained from this study highlights that $D$. esculentum may be beneficial for the prevention or treatment of $\mathrm{AD}$.

Received: 1 August 2021; Accepted: 26 November 2021

Published online: 10 December 2021

\section{References}

1. Ashraf, G. M. et al. Recent updates on the association between Alzheimer's disease and vascular dementia. Med. Chem. 12, 226-237. https://doi.org/10.2174/1573406411666151030111820 (2016).

2. Collaborators, G. B. D. D. Global, regional, and national burden of Alzheimer's disease and other dementias, 1990-2016: A systematic analysis for the Global Burden of Disease Study 2016. Lancet Neurol. 18, 88-106. https://doi.org/10.1016/S1474-4422(18) 30403-4 (2019).

3. Ferreira-Vieira, T. H., Guimaraes, I. M., Silva, F. R. \& Ribeiro, F. M. Alzheimer's disease: Targeting the cholinergic system. Curr. Neuropharmacol. 14, 101-115. https://doi.org/10.2174/1570159x13666150716165726 (2016).

4. Wilcock, G. K., Esiri, M. M., Bowen, D. M. \& Smith, C. C. Alzheimer's disease. Correlation of cortical choline acetyltransferase activity with the severity of dementia and histological abnormalities. J. Neurol. Sci. 57, 407-417. https://doi.org/10.1016/0022510x(82)90045-4 (1982).

5. Serrano-Pozo, A., Frosch, M. P., Masliah, E. \& Hyman, B. T. Neuropathological alterations in Alzheimer disease. Cold Spring Harb. Perspect. Med. 1, a006189. https://doi.org/10.1101/cshperspect.a006189 (2011).

6. O’Brien, R. J. \& Wong, P. C. Amyloid precursor protein processing and Alzheimer's disease. Annu. Rev. Neurosci. 34, 185-204. https://doi.org/10.1146/annurev-neuro-061010-113613 (2011).

7. Moussa-Pacha, N. M., Abdin, S. M., Omar, H. A., Alniss, H. \& Al-Tel, T. H. BACE1 inhibitors: Current status and future directions in treating Alzheimer's disease. Med. Res. Rev. 40, 339-384. https://doi.org/10.1002/med.21622 (2020).

8. Goedert, M. \& Spillantini, M. G. A century of Alzheimer's disease. Science 314(5800), 777-781 (2006).

9. Citron, M. et al. Evidence that the 42 - and 40 -amino acid forms of amyloid $\beta$ protein are generated from the $\beta$-amyloid precursor protein by different protease activities. Proc. Natl. Acad. Sci. U.S.A. 93, 13170-13175 (1996).

10. Casey, D. A., Antimisiaris, D. \& O'Brien, J. Drugs for Alzheimer's disease: Are they effective?. P T 35, 208-211 (2010).

11. Wohlfarth, C. \& Efferth, T. Natural products as promising drug candidates for the treatment of hepatitis B and C. Acta Pharmacol. Sin. 30, 25-30. https://doi.org/10.1038/aps.2008.5 (2009).

12. Zannah, F., Amin, M., Suwono, H. \& Lukiati, B. Phytochemical screening of Diplazium esculentum as medicinal plant from Central Kalimantan, Indonesia. AIP Conf. Proc. 1844, 050001. https://doi.org/10.1063/1.4983439 (2017).

13. Das, B., Paul, T., Apte, K. G., Chauhan, R. \& Saxena, R. C. Evaluation of antioxidant potential \& quantification of polyphenols of Diplazium esculentum Retz. with emphasis on its HPTLC chromatography. J. Pharm. Res. 6, 93-100. https://doi.org/10.1016/j. jopr.2012.11.020 (2013).

14. Zihad, S. M. N. K. et al. Nutritional value, micronutrient and antioxidant capacity of some green leafy vegetables commonly used by southern coastal people of Bangladesh. Heliyon 5, e02768. https://doi.org/10.1016/j.heliyon.2019.e02768 (2019).

15. Junejo, J. A. et al. Exploration of antioxidant, antidiabetic and hepatoprotective activity of Diplazium esculentum: A wild edible plant from North Eastern India. Future J. Pharm. Sci. 4, 93-101. https://doi.org/10.1016/j.fjps.2017.10.005 (2018).

16. Amit, S., Sunil, K., Sp, B. \& Arvind, N. Antibacterial activity of Diplazium esculentum (Retz.) Sw. Pharmacogn. J. 3, 77-79. https:// doi.org/10.5530/pj.2011.21.14 (2011).

17. Roy, S., Dutta, S. \& Chaudhuri, T. K. In vitro assessment of anticholinesterase and NADH oxidase inhibitory activities of an edible fern, Diplazium esculentum. J. Basic Clin. Physiol. Pharmacol. 26, 395-401. https://doi.org/10.1515/jbcpp-2014-0100 (2015).

18. Duffy, J. B. GAL4 system in Drosophila: A fly geneticist's Swiss army knife. Genesis 34, 1-15. https://doi.org/10.1002/gene.10150 (2002).

19. Quon, D. et al. Formation of beta-amyloid protein deposits in brains of transgenic mice. Nature 352, 239-241. https://doi.org/10. 1038/352239a0 (1991).

20. Tsuda, L. \& Lim, Y.-M. in Drosophila Models for Human Diseases (ed Yamaguchi, M.) 25-40 (Springer, Singapore, 2018).

21. Bagchi, S. et al. In-vitro blood-brain barrier models for drug screening and permeation studies: an overview. Drug Des. Dev. Ther. 13, 3591-3605. https://doi.org/10.2147/DDDT.S218708 (2019).

22. Green, K. D., Fosso, M. Y. \& Garneau-Tsodikova, S. Multifunctional donepezil analogues as cholinesterase and BACE1 inhibitors. Molecules https://doi.org/10.3390/molecules23123252 (2018). 
23. Huovila, A.-P.J., Turner, A. J., Pelto-Huikko, M., Kärkkäinen, I. \& Ortiz, R. M. Shedding light on ADAM metalloproteinases. Trends Biochem. Sci. 30, 413-422. https://doi.org/10.1016/j.tibs.2005.05.006 (2005).

24. D’Onofrio, G. et al. Phytochemicals in the treatment of Alzheimer's disease: A systematic review. Curr. Drug Targets 18, 1487-1498. https://doi.org/10.2174/1389450117666161102121553 (2017).

25. Tungmunnithum, D., Thongboonyou, A., Pholboon, A. \& Yangsabai, A. Flavonoids and other phenolic compounds from medicinal plants for pharmaceutical and medical aspects: An overview. Medicines (Basel) https://doi.org/10.3390/medicines5030093 (2018).

26. Akter, S., Hossain, M. M., Ara, I. \& Akhtar, P. Investigation of in vitro antioxidant, antimicrobial and cytotoxic activity of Diplazium esculentum (Retz). Sw. IJAPBC 3, 723-733 (2014).

27. Umikalsom, Y., Grayer-Barkmeijer, R. J. \& Harborne, J. B. A comparison of the flavonoids in Athyriaceae and Aspleniaceae. Biochem. Syst. Ecol. 22, 587-594. https://doi.org/10.1016/0305-1978(94)90071-X (1994).

28. Kumar, S. \& Pandey, A. K. Chemistry and biological activities of flavonoids: An overview. Sci. World J. 2013, 162750. https://doi. org/10.1155/2013/162750 (2013).

29. Ademosun, A. O., Oboh, G., Bello, F. \& Ayeni, P. O. Antioxidative properties and effect of quercetin and its glycosylated form (Rutin) on acetylcholinesterase and butyrylcholinesterase activities. J. Evid. Based Complement. Altern. Med. 21, NP11-NP17. https://doi.org/10.1177/2156587215610032 (2016).

30. Shimmyo, Y., Kihara, T., Akaike, A., Niidome, T. \& Sugimoto, H. Flavonols and flavones as BACE-1 inhibitors: Structure-activity relationship in cell-free, cell-based and in silico studies reveal novel pharmacophore features. Biochim. Biophys. Acta 1780, 819-825. https://doi.org/10.1016/j.bbagen.2008.01.017 (2008).

31. Descamps, O. et al. AbetaPP-selective BACE inhibitors (ASBI): Novel class of therapeutic agents for Alzheimer's disease. J. Alzheimers Dis. 37, 343-355. https://doi.org/10.3233/JAD-130578 (2013).

32. Wang, X. et al. Effects of curcuminoids identified in rhizomes of Curcuma longa on BACE-1 inhibitory and behavioral activity and lifespan of Alzheimer's disease Drosophila models. BMC Complement. Altern. Med. 14, 88. https://doi.org/10.1186/1472-6882-1488 (2014).

33. Huang, P., Zheng, N., Zhou, H.-B. \& Huang, J. Curcumin inhibits BACE1 expression through the interaction between ER $\beta$ and NFkB signaling pathway in SH-SY5Y cells. Mol. Cell. Biochem. 463, 161-173. https://doi.org/10.1007/s11010-019-03638-0 (2020).

34. Tennessen, J. M., Baker, K. D., Lam, G., Evans, J. \& Thummel, C. S. The Drosophila estrogen-related receptor directs a metabolic switch that supports developmental growth. Cell Metab. 13, 139-148. https://doi.org/10.1016/j.cmet.2011.01.005 (2011).

35. Hetru, C. \& Hoffmann, J. A. NF-kappaB in the immune response of Drosophila. Cold Spring Harb. Perspect. Biol. 1, a000232. https:// doi.org/10.1101/cshperspect.a000232 (2009).

36. Ou-yang, Z. et al. Pharmacokinetic study of rutin and quercetin in rats after oral administration of total flavones of mulberry leaf extract. Rev. Bras. 23, 776-782. https://doi.org/10.1590/S0102-695X2013000500009 (2013).

37. Ono, K. et al. Potent anti-amyloidogenic and fibril-destabilizing effects of polyphenols in vitro: Implications for the prevention and therapeutics of Alzheimer's disease. J. Neurochem. 87, 172-181. https://doi.org/10.1046/j.1471-4159.2003.01976.x (2003).

38. Kong, Y. et al. Quercetin ameliorates A $\beta$ toxicity in Drosophila AD model by modulating cell cycle-related protein expression. Oncotarget 7, 67716-67731. https://doi.org/10.18632/oncotarget.11963 (2016).

39. Bartolini, M., Bertucci, C., Cavrini, V. \& Andrisano, V. beta-Amyloid aggregation induced by human acetylcholinesterase: Inhibition studies. Biochem. Pharmacol. 65, 407-416. https://doi.org/10.1016/s0006-2952(02)01514-9 (2003).

40. Butterfield, D. A., Swomley, A. M. \& Sultana, R. Amyloid beta-peptide (1-42)-induced oxidative stress in Alzheimer disease: Importance in disease pathogenesis and progression. Antioxid. Redox Signal. 19, 823-835. https://doi.org/10.1089/ars.2012.5027 (2013)

41. Liang, N. \& Kitts, D. D. Antioxidant property of coffee components: Assessment of methods that define mechanisms of action. Molecules 19, 19180-19208. https://doi.org/10.3390/molecules191119180 (2014).

42. Carmine-Simmen, K. et al. Neurotoxic effects induced by the Drosophila amyloid-beta peptide suggest a conserved toxic function. Neurobiol. Dis. 33, 274-281. https://doi.org/10.1016/j.nbd.2008.10.014 (2009).

43. Chakraborty, R. et al. Characterization of a Drosophila Alzheimer's disease model: Pharmacological rescue of cognitive defects. PLoS ONE 6, e20799. https://doi.org/10.1371/journal.pone.0020799 (2011).

44. Bergkvist, L., Sandin, L., Kågedal, K. \& Brorsson, A. C. A $\beta$ PP processing results in greater toxicity per amount of A $\beta 1-42$ than individually expressed and secreted A $\beta 1-42$ in Drosophila melanogaster. Biol. Open 5, 1030-1039. https://doi.org/10.1242/bio. 017194 (2016).

45. Iijima-Ando, K. \& Iijima, K. Transgenic Drosophila models of Alzheimer's disease and tauopathies. Brain Struct. Funct. 214, 245-262. https://doi.org/10.1007/s00429-009-0234-4 (2010).

46. Liu, Q. F. et al. Coriandrum sativum suppresses A $\beta 42$-induced ROS increases, glial cell proliferation, and ERK activation. Am. J. Chin. Med. 44, 1325-1347. https://doi.org/10.1142/s0192415x16500749 (2016).

47. Ainsworth, E. A. \& Gillespie, K. M. Estimation of total phenolic content and other oxidation substrates in plant tissues using Folin-Ciocalteu reagent. Nat. Protoc. 2, 875-877. https://doi.org/10.1038/nprot.2007.102 (2007).

48. Thuphairo, K., Sornchan, P. \& Suttisansanee, U. Bioactive compounds, antioxidant activity and inhibition of key enzymes relevant to Alzheimer's disease from sweet pepper (Capsicum annuum) extracts. Prev Nutr Food Sci 24, 327-337. https://doi.org/10.3746/ pnf.2019.24.3.327 (2019).

49. Sritalahareuthai, V., Temviriyanukul, P., On-Nom, N., Charoenkiatkul, S. \& Suttisansanee, U. Phenolic profiles, antioxidant, and inhibitory activities of Kadsura heteroclita (Roxb.) Craib and Kadsura coccinea (Lem.) A.C. Sm. Foods https://doi.org/10.3390/ foods9091222 (2020).

50. Benzie, I. F. \& Strain, J. J. The ferric reducing ability of plasma (FRAP) as a measure of "antioxidant power": the FRAP assay. Anal. Biochem. 239, 70-76. https://doi.org/10.1006/abio.1996.0292 (1996).

51. Fukumoto, L. R. \& Mazza, G. Assessing antioxidant and prooxidant activities of phenolic compounds. J. Agric. Food Chem. 48, 3597-3604 (2000).

52. Prior, R. L. et al. Assays for hydrophilic and lipophilic antioxidant capacity (oxygen radical absorbance capacity (ORAC(FL)) of plasma and other biological and food samples. J. Agric. Food Chem. 51, 3273-3279. https://doi.org/10.1021/jf0262256 (2003).

53. Temviriyanukul, P. et al. Comparison of phytochemicals, antioxidant, and in vitro anti-Alzheimer properties of twenty-seven Morus spp. cultivated in Thailand. Molecules https://doi.org/10.3390/molecules25112600 (2020).

54. Jung, H. A. et al. Anti-Alzheimer and antioxidant activities of Coptidis Rhizoma alkaloids. Biol. Pharm. Bull. 32, 1433-1438 (2009).

55. Temviriyanukul, P. et al. The effect of sacred lotus (Nelumbo nucifera) and Its mixtures on phenolic profiles, antioxidant activities, and inhibitions of the key enzymes relevant to Alzheimer's disease. Molecules https://doi.org/10.3390/molecules25163713 (2020).

56. Jantrapirom, S., Lo Piccolo, L., Yoshida, H. \& Yamaguchi, M. A new Drosophila model of Ubiquilin knockdown shows the effect of impaired proteostasis on locomotive and learning abilities. Exp. Cell Res. 362, 461-471. https://doi.org/10.1016/j.yexcr.2017. 12.010 (2018).

57. Luheshi, L. M. et al. Sequestration of the Abeta peptide prevents toxicity and promotes degradation in vivo. PLoS Biol. 8, e1000334. https://doi.org/10.1371/journal.pbio.1000334 (2010).

58. Rogers, I. et al. Ageing increases vulnerability to a 342 toxicity in Drosophila. PLoS ONE 7, e40569. https://doi.org/10.1371/journ al.pone.0040569 (2012). 
Acknowledgements

We thank Dr. Kanchana Pruesapan, Plant Varieties Protection Division, Department of Agriculture, Bangkok for sample management and registration. We also thank Ms. Pisamai Ting for training T.K. on Drosophila studies.

\title{
Author contributions
}

Conceptualization, P.T., D.T. and U.S.; methodology, T.K., U.S. and P.T.; validation, T.K., W.I., and J.K.; formal analysis, T.K.; investigation, T.K.,J.K., and U.S.; resources, T.K. and P.P.; data curation, T.K.; writing-original draft preparation, U.S., P.P. and P.T.; writing-review and editing, U.S., D.T., B.C., S.P. and P.T.; visualization, T.K. and W.I.; supervision, U.S., D.T. and P.T.; funding acquisition, T.K., S.P., P.P. and P.T. All authors have read and agree to the published version of the manuscript.

\section{Funding}

This study was funded by National Research Council of Thailand (NRCT) for FY2017 Thesis grant and graduate studies of Mahidol University Alumni Association to T.K., and partially supported by Grants-in-Aid from JSPS Core to-Core Program B. Asia-Africa Science Platforms to S.P., P.P. and P.T.

\section{Competing interests}

The authors declare no competing interests.

\section{Additional information}

Supplementary Information The online version contains supplementary material available at https://doi.org/ 10.1038/s41598-021-03142-w.

Correspondence and requests for materials should be addressed to P.T.

Reprints and permissions information is available at www.nature.com/reprints.

Publisher's note Springer Nature remains neutral with regard to jurisdictional claims in published maps and institutional affiliations.

\begin{abstract}
Open Access This article is licensed under a Creative Commons Attribution 4.0 International License, which permits use, sharing, adaptation, distribution and reproduction in any medium or format, as long as you give appropriate credit to the original author(s) and the source, provide a link to the Creative Commons licence, and indicate if changes were made. The images or other third party material in this article are included in the article's Creative Commons licence, unless indicated otherwise in a credit line to the material. If material is not included in the article's Creative Commons licence and your intended use is not permitted by statutory regulation or exceeds the permitted use, you will need to obtain permission directly from the copyright holder. To view a copy of this licence, visit http://creativecommons.org/licenses/by/4.0/.
\end{abstract}

(C) The Author(s) 2021 